Sonderdruck aus

J USTUS LIEBIGS AN NALEN DER CHEM IE Liebigs Ann. Chem. 737, 119-127 (1970)

\title{
Eine Farbreaktion der 1-Benzoyl-3.4-dihydro-isochinoline
}

von Herbert Budzikiewicz und Uwe Krüger

Aus dem Institut der Gesellschaft für Molekularbiologische Forschung, D-3301 Stöckheim sowie Wulf-Dieter Sasse und Wolfgang Wiegrebe

Aus dem Institut für Pharmazeutische Technologie der Technischen Universität, D-33 Braunschweig

(C) Verlag Chemie, GmbH, Weinheim/Bergstr. 1970 


\title{
Eine Farbreaktion der 1-Benzoyl-3.4-dihydro-isochinoline
}

\author{
von Herbert Budzikiewicz und Uwe Krüger
}

Aus dem Institut der Gesellschaft für Molekularbiologische Forschung, D-3301 Stöckheim sowie Wulf-Dieter Sasse und Wolfgang Wiegrebe

Aus dem Institut für Pharmazeutische Technologie der Technischen Universität,

D-33 Braunschweig

Eingegangen am 15. Januar 1970

Die tiefgrüne Farbe, die beim Erhitzen von 1-[3.4-Dimethoxy-benzoyl]-6.7-dimethoxy3-methyl-3.4-dihydro-isochinolin ( $2 ; \mathrm{R}^{1}=\mathrm{CH}_{3}$ ) mit Acetanhydrid entsteht, ist auf 1-[3.4-Dimethoxy-phenyl]-8.9-dimethoxy-5-methyl-2.3.5.6-tetrahydro-pyrrolo[2.1-a]isochinolin-3-on (8) zurückzuführen.

\section{A Colour Reaction of 1-Benzoyl-3,4-dihydroisoquinolines}

Heating of 1-[3,4-dimethoxy-benzoyl]-6,7-dimethoxy-3-methyl-3,4-dihydroisoquinoline (2: $\mathbf{R}^{1}=\mathrm{CH}_{3}$ ) with acetic anhydride gives an intense green colour which is due to 1-[3,4-dimethoxy-phenyl]-8,9-dimethoxy-5-methyl-2,3,5,6-tetrahydro-pyrrolo[2,1-a]isoquinoline-3-one (8).

Im Zusammenhang mit Arbeiten über Reaktionen der Tetrahydroisochinolyl-phenylcarbinole1) 1 untersuchten wir eine Farbreaktion der 1-Benzoyl-3.4-dihydro-isochinoline 2, da Verbindungen dieses Typs Vorstufen für Derivate von 1 sind 2).

Es ist bekannt ${ }^{3}$, daß die Lösung von 3.4-Dihydro-papaveraldin $\left(2: R^{1}=H\right)$ in Acetanhydrid beim Kochen grün wird. Wir fanden, daß andere 1-Benzoyl-3.4-dihydroisochinoline 2 ebenso reagieren ${ }^{2)}$.

Die beim Kochen von $2\left(R^{1}=H\right)$ mit überschüssigem Acetanhydrid anfangs durch Salzbildung gelbbraune Lösung färbte sich schnell tiefgrün $\left(\lambda_{\max }=730 \mathrm{~nm}\right)$. Diese Farbe blieb in Acetanhydrid etwa $4 \mathrm{Stdn}$. bestehen und ging schließlich in Braunrot über. Chromatographische Untersuchungen zeigten, daß mindestens 20 Verbindungen verschiedener Farbe entstanden waren.

Wir trennten das Gemisch im Stas-Otto-Gang nach den Angabẹn auf S. 125. Als Hauptprodukt isolierten wir aus den in Äther schwerlöslichen Neutralstoffen schwach

1) W. Wiegrebe und W. Awe, Arch. Pharmaz. 296, 807 (1963).

2) W. Wiegrebe, Arch. Pharmaz. 297, 362 (1964).

3) J. S. Buck, R. D. Haworth und W. H. Perkin jr., J. chem. Soc. [London] 125, 2176 (1924). 
gelbe Nadeln vom Schmp. $172^{\circ}$. Die Elementaranalyse lieferte die Formel $\mathrm{C}_{22} \mathrm{H}_{25} \mathrm{NO}_{7}$ (2: $\mathbf{R}^{1}=\mathbf{H}+1$ Mol. Essigsäure). Der Neutralstoffcharakter deutete darauf hin, daß die gegenüber dem Ausgangsmaterial hinzugekommenen 2 C-Atome eine AcetamidGruppierung gebildet hatten, was eine Ringsprengung des Heterocyclus wahrscheinlich machte.

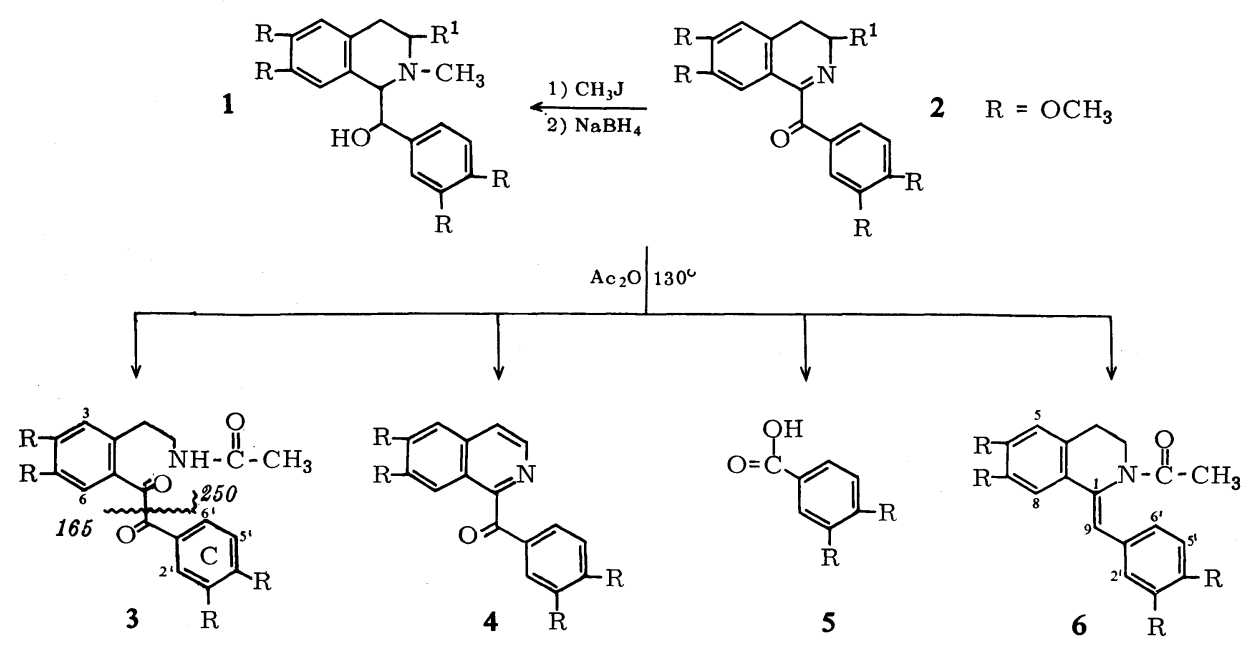

Im IR-Spektrum (KBr) traten Banden bei 1635 und $3310 \mathrm{~cm}^{-1}$ (Amid) und $1660 \mathrm{~cm}^{-1}$ (Arylketon) auf. Das NMR-Spektrum $\left(\mathrm{CDCl}_{3}\right)$ bewies, daß der $\operatorname{Ring} \mathrm{C}$ in $2\left(\mathrm{R}^{1}=\mathrm{H}\right)$ nicht substituiert worden war. Im Bereich der aromatischen Protonen traten Signale auf bei $\tau=$ $2.55\left(\mathrm{~d}, \mathrm{~J}_{m}=2.0 \mathrm{~Hz} ; \mathrm{H}-2^{\prime}\right), 2.68\left(\mathrm{dd}, \mathrm{J}_{m}=2.0 \mathrm{~Hz}, \mathrm{~J}_{o}=9 \mathrm{~Hz} ; \mathrm{H}-6^{\prime}\right), 3.22\left(\mathrm{~d}, \mathrm{~J}_{o}=9 \mathrm{~Hz}\right.$; H-5'), 3.00 (s; H-6), 3.26 (s; H-3). Weiter ist ein Singulett bei $\tau=8.10$ (Acetamid-Gruppe) zu beobachten, sowie ein Signal bei $\tau=3.96$ (breites Singulett; NH), das beim Austausch mit $\mathrm{D}_{2} \mathrm{O}$ verschwindet. $\mathrm{D}_{2} \mathrm{O}$-Austausch bewirkt auch, daß zwei Methylen-Signale $(\tau=6.84$ bzw. 6.51) als Tripletts erscheinen, während vor dem Austausch das Niederfeldsignal durch zusätzliche Kopplung mit dem NH-Proton zu einem Quartett aufgespalten war $(\mathrm{J}=7 \mathrm{~Hz})$.

Für die Öffnung des heterocyclischen Ringes und damit die Bildung eines Benzil-Derivates spricht auch die geringe Intensität des Molekül-Ions (1\%; vgl. z. B. das Massenspektrum von 4, bei dem $\mathrm{M}^{\oplus} 100 \%$ rel. Intensität ausmacht). Eines der Hauptspaltprodukte, das bei $\mathrm{m} / \mathrm{e}=165(36 \%)$ auftritt, ist das 3.4-Dimethoxy-benzoyl-Kation (vgl. Formel 3), das das Vorhandensein des unveränderten Ringes $C$ bestätigt. Das Komplementärfragment $(\mathrm{m} / \mathrm{e}=$ $250 ; 12 \%)$ kann seinerseits unter Abspaltung von Keten ${ }^{4)}$ weiter zerfallen (m/e $\left.=208 ; 100 \%\right)$, wie es für die Anwesenheit einer Acetamid-Gruppe zu erwarten ist.

4) H. Budzikiewicz, C. Djerassi und D. H. Williams, Mass Spectrometry of Organic Compounds, S. 336f., Holden Day Inc., San Francisco 1967. 
Diese Befunde zeigen, daß als Hauptprodukt 2-[3-Acetamino-äthyl]-4.5.3'.4'-tetramethoxy-benzil (3) entstanden war. Der chemische Beweis für diese Struktur wurde durch Erhitzen von 3 mit konz. Salzsäure erbracht, wobei sich $2\left(\mathbf{R}^{1}=\mathbf{H}\right)$ durch Verseifung der Acetamid-Gruppierung und säurekatalysierten Ringschluß zurückbildete.

In der Basen-Phase des Reaktionsgemisches aus $2\left(\mathrm{R}^{1}=\mathrm{H}\right)$ wurde neben dem Ausgangsmaterial dessen Dehydrierungsprodukt Papaveraldin (4) nachgewiesen.

Aus der Fraktion der sauren bzw. phenolischen Reaktionsprodukte wurde 3.4-Dimethoxy-benzoesäure (5) erhalten und direkt sowie als Methylester durch Vergleich mit authentischen Substanzen identifiziert.

Die Fraktion der ätherlöslichen Neutralstoffe enthielt eine farblose Verbindung (Schmp. 202 ${ }^{\circ}$ ) der Zusammensetzung $\mathrm{C}_{22} \mathrm{H}_{25} \mathrm{NO}_{5}$, deren UV-Spektrum (Methanol) folgende Maxima aufwies: $\lambda_{\max }(\log \varepsilon)=220$ (4.45), $332 \mathrm{~nm}$ (4.43). Das IR-Spektrum $(\mathrm{KBr})$ zeigte nur eine Carbonyl-Bande bei $1633 \mathrm{~cm}^{-1}$ (Amid). Dieser Befund und das Ergebnis der Elementaranalyse zeigen, daß eine Reduktion stattgefunden haben muß.

Der Mol-Peak des Massenspektrums liegt bei $\mathrm{m} / \mathrm{e}=383$ (100\%). Durch Verlust eines Methyl-Radikals entsteht das Fragment $\mathrm{m} / \mathrm{e}=368(18 \%)$, das seinerseits Keten abspaltet und $\mathrm{zu} \mathrm{m} / \mathrm{e}=326(83 \%)$ führt. Der Mol.-Peak verliert ebenfalls Keten $\left.{ }^{4}\right)(\mathrm{m} / \mathrm{e}=341 ; 39 \%)$; außerdem kann ein Acyl-Rest abgespalten werden ( $/ \mathrm{e}=340 ; 64 \%$ ). Das restliche Spektrum ist uncharakteristisch. Das NMR-Spektrum zeigt die in Tab. 1 angegebenen Signale.

Tabelle 1. $\tau$-Werte, Multiplizitäten und Zuordnungen der NMR-Signale $\left(\mathrm{CDCl}_{3}\right.$; Tetramethylsilan als innerer Standard) von 1-[3.4-Dimethoxy-benzyliden]-2-acetyl-6.7-dimethoxy1.2.3.4-tetrahydro-isochinolin (6), $N$-Acetyl-1.2.3.4-tetrahydro-papaverin (7) und 1-[3.4-Dimethoxy-phenyl]-8.9-dimethoxy-5-methyl-2.3.5.6-tetrahydro-pyrrolo[2.1-a]isochinolin-3-on (8)

\begin{tabular}{llcc}
\hline & \multicolumn{1}{c}{6} & 7 & 8 \\
\hline $\mathrm{H}-3(\mathrm{a})$ & $7.40(\mathrm{dd})$ & $7.34(\mathrm{dd})$ & - \\
$\mathrm{H}-3(\mathrm{~b})$ & $5.09(\mathrm{dd})$ & $5.28(\mathrm{dd})$ & - \\
$\mathrm{H}-4$ & $6.89(\mathrm{dd})$ & $7.0(\mathrm{~m})$ & - \\
$\mathrm{H}-5$ & $3.44(\mathrm{~s})$ & - & $5.41(\mathrm{q})$ \\
$\mathrm{H}-6$ & - & - & 7.39 \\
$\mathrm{H}-8$ & $3.53(\mathrm{~s})$ & - & - \\
$\mathrm{H}-9$ & $3.02(\mathrm{~s})$ & - & - \\
$\mathrm{H}-2^{\prime}$ & $3.08\left(\mathrm{~d}, \mathrm{~J}_{m}=2 \mathrm{~Hz}\right)$ & - & - \\
$\mathrm{H}-5^{\prime}$ & $3.30\left(\mathrm{~d}, \mathrm{~J}_{o}=9 \mathrm{~Hz}\right)$ & - & $3.48\left(\mathrm{~d}, \mathrm{~J}_{o}=8.5 \mathrm{~Hz}\right)$ \\
$\mathrm{H}-6^{\prime}$ & $3.10\left(\mathrm{dd}, \mathrm{J}^{\prime}=2 \mathrm{~Hz}, \mathrm{~J}=9 \mathrm{~Hz}\right)$ & - & - \\
$\mathrm{H}_{3} \mathrm{C}-\mathrm{CO}$ & $8.26(\mathrm{~s})$ & $8.37+7.89(\mathrm{~s} ; 3 \mathrm{H})$ & -
\end{tabular}


Auf Grund der voranstehenden Befunde handelt es sich bei der Substanz $\mathrm{C}_{22} \mathrm{H}_{25} \mathrm{NO}_{5}$ um 1-[3.4-Dimethoxy-benzyliden]-2-acetyl-6.7-dimethoxy-1.2.3.4-tetrahydro-isochinolin (6).

Baxter und $S_{w a n}{ }^{5)}$ haben diese Verbindung durch Acetylierung von 3.4-Dihydro-papaverin (2: $\mathrm{R}^{1}=\mathrm{H} ; \mathrm{CH}_{2}$ statt $\mathrm{CO}$ ) erhalten, das auch wir als $\mathrm{Zwischenprodukt} \mathrm{annehmen} \mathrm{möchten;}$ sie geben allerdings den Schmp. $193^{\circ}$ für 6 an. Nach ihrer Vorschrift $\left.{ }^{5}\right)$ hergestelltes 6 war mit unserer Verbindung identisch.

Im NMR-Spektrum von 6 fiel auf, daß die Protonen an C-3 einen Unterschied von $2.3 \mathrm{ppm}$ in der chemischen Verschiebung aufweisen. Es ist bekannt, daß die anisotrope Verschiebung durch die Amidgruppe stark von der räumlichen Stellung der Substituenten an dem dem $\mathbf{N}$ benachbarten C-Atom abhängig ist ${ }^{6}$. Um zu prüfen, ob diese Erklärung auch für das NMR-Spektrum von 6 zutrifft, haben wir $N$-Acetyl-1.2.3.4-tetrahydro-papaverin (7; 1.9-Dihydro-6) NMR-spektroskopisch untersucht (vgl. Tab. 1). Die Lage der Protonen H-3 (a), H-3 (b) und H-4 ist analog. H-1 tritt bei $\tau=4.43$ als Doppeldublett auf. Die Aufgliederung des Signals der Acetylgruppe in zwei Singuletts erklären wir mit Rotationshinderung um die $\mathrm{C}-\mathrm{N}$-Amid-Bindung. Dementsprechend fallen die Signale beim Erhitzen auf $140^{\circ}$ in Tetrachloräthan- $d_{2}$ zu einem Singulett zusammen.

Aus der 6 enthaltenden Phase ließen sich außerdem zwei weinrote, äußerst labile Verbindungen $\left(A\right.$ und $B$ ) isolieren. Beide Substanzen zersetzten sich auch unter $\mathrm{N}_{2}$ bei dem Versuch, sie durch präparative Schichtchromatographie weiter zu reinigen und konnten daher nicht identifiziert werden. Da sich die Lösung des grünen Rohproduktes in Wasser/Methanol bereits in der Kälte innerhalb $30 \mathrm{Min}$. rot färbt, nehmen wir an, da $B A$ und $B$ Folgeprodukte sind.

Die im Formelbild auf S. 120 dargestellten Verbindungen zeigen, daß bei der Umsetzung von $2\left(\mathrm{R}^{1}=\mathrm{H}\right)$ mit Acetanhydrid acetolytische $(2 \rightarrow 3)$, dehydrierende $(2 \rightarrow 4)$, oxydierende $(2 \rightarrow 5)$ und reduzierende $(2 \rightarrow 6)$ Prozesse nebeneinander ablaufen.

$\mathrm{Da}$ das grüne Reaktionsprodukt aus $2\left(\mathrm{R}^{1}=\mathrm{H}\right)$ labil ist, haben wir die gleiche Umsetzung auf $2\left(\mathrm{R}^{1}=\mathrm{CH}_{3}\right)$ übertragen. Da außerdem mehrere, $N$-acetylierte Produkte auftreten, wurde geprüft, ob die Grünfärbung auch von $C$-3-alkylierten 1-Benzoyl-3.4-dihydro-isochinolinen gegeben wird. Wir hatten nämlich beobachtet, daß die Fähigkeit des Isochinolin-Stickstoffs, nucleophil zu reagieren, durch $C$-3-Alkylierung sinkt7).

Dazu stellten wir 3-Methyl-3.4-dihydro-papaveraldin (2: $\left.\mathbf{R}^{1}=\mathrm{CH}_{3}\right)$ durch Oxydation von 3-Methyl-3.4-dihydro-papaverin8) (2: $\mathrm{R}^{1}=\mathrm{CH}_{3} ; \mathrm{CH}_{2}$ statt $\left.\mathrm{CO}\right)$ mit

5) I. Baxter und G. A. Swan, J. chem. Soc. [London] 1965, 4014.

6) 6a) H. Paulsen und K. Todt, Angew. Chem. 78, 943 (1966). - 6b) U. Krüger und H. Hettler,

Z. Elektrochem., Ber. Bunsenges. physik. Chem. 73, 15 (1969).

7) W. Wiegrebe, Arch. Pharmaz. 300, 332, 712 (1967).

8) 8a) E. Merck, Dtsch. Reichs-Pat. 550122 [C. 1931 II, 1196]. - 8b) W. Wiegrebe, Arch. Pharmaz. 300, 716 (1967); dort weitere Lit. 
Selendioxid her und erhielten bei der Umsetzung von $2\left(\mathbf{R}^{1}=\mathrm{CH}_{3}\right)$ mit Acetanhydrid ebenfalls eine tiefgrüne Lösung $\left(\lambda_{\max }=730 \mathrm{~nm}\right)$. Dieser Farbstoff war jedoch gegen Hydrolyse beständig: Auch nach 1 Tag in Methanol/verd. Schwefelsäure bei $5^{\circ}$ war die Grünfärbung unverändert. Wir gewannen den grünen Farbstoff direkt durch Schichtchromatographie des Rohproduktes in kleinen, violett schimmernden Plättchen vom Schmp. $226-228^{\circ}$.

Das UV-Spektrum (Methanol) zeigt Maxima (log $\varepsilon$ ) bei 233 (4.29), 335 (3.98), 440 (3.62) und $730 \mathrm{~nm}$ (4.07). Das Massenspektrum dieser Verbindung zeigt $\mathrm{M}^{\oplus}$ bei $\mathrm{m} / \mathrm{e}=395(100 \%)$. Fragmentierung durch Verlust von ${ }^{\circ} \mathrm{CH}_{3}$ führt $\mathrm{zu} \mathrm{m} / \mathrm{e}=380$ (65\%). Offensichtlich enthält das Molekül keine Bindungen, deren Spaltung energetisch besonders bevorzugt ist. Obwohl die Verbindung ein Neutralstoff ist, spaltet sie kein Keten ab; es liegt somit keine freie Acetamid-Gruppe vor. Das IR-Spektrum (KBr) zeigt eine Bande bei $1680 \mathrm{~cm}^{-1}$. Wir schließen aus diesen Befunden auf eine Acetamid-Gruppierung, deren $\alpha$-C-Atom eine weitere Bindung eingegangen ist (das NMR-Spektrum zeigt kein Methyl-Signal einer Acetylgruppe). Diese Bindung konnte nicht zum C-6' der Benzoylgruppe geknüpft worden sein, denn das NMRSpektrum zeigte, daß im Bereich aromatischer Protonen nicht nur Singuletts auftreten. Bei $\tau=3.48$ tritt das Dublett von $\mathrm{H}^{-5}{ }^{\prime}$ auf (Tab. 1); durch sukzessive Zugabe von Deuterobenzol bis zu 50 Vol.- \% waren auch H-2' als Dublett mit $\mathrm{J}_{m}=2 \mathrm{~Hz}$ und die Niederfeldhälfte des Doppeldubletts von H-6' zu erkennen. Die Methyl-Gruppe am C-5 erscheint als Dublett bei $\tau=8.94(\mathrm{~J}=7 \mathrm{~Hz})$. H-5 tritt als Quintett bei $\tau=5.41$ auf: Das Signal wird durch die Methylgruppe zum Quartett und durch etwa gleichgroße Kopplung mit dem syn-ständigen Proton H-6 zum Quintett aufgespalten. Die Protonen an C-2 erscheinen als schlecht aufgelöstes Dublett bei $\tau=6.8$; vermutlich handelt es sich um die verbreiterten Innenlinien eines AB-Systems.

Aufgrund der spektroskopischen Befunde nahmen wir an, daß es sich bei den blauvioletten Kristallen um 1-[3.4-Dimethoxy-phenyl]-8.9-dimethoxy-5-methyl-2.3.5.6-tetrahydro-pyrrolo[2.1-a]isochinolin-3-on (8) handelt.

Versuche, 8 katalytisch oder mit Hydriden zu hydrieren, führten zu unüberschaubaren Gemischen. Wir oxydierten daher 8 mit Permanganat in Pyridin/Wasser. Dabei sollten als Beweis für die Verknüpfung des $\alpha$-C-Atoms der Amid-Gruppierung mit dem Benzyl-C-Atom zum Lactam 3.4-Dimethoxy-acetophenon (9) und eventuell - als dessen Folgeprodukt - 3.4-Dimethoxy-benzoesäure (5) auftreten.

In früheren Versuchen ${ }^{9)}$ hatten wir allerdings festgestellt, daß Acetophenon unter diesen Bedingungen stabil ist.

Oxydativer Abbau von 8 führte zu zahlreichen Produkten. Nach Vortrennung im Stas-Otto-Gang fanden wir in der Säure-Fraktion u. a. eine Substanz, die mit 2.4-Dinitro-phenylhydrazin eine positive Carbonylgruppen-Reaktion gab10). Da 9 nicht vorhanden war, vermuteten wir, da $\beta \beta$-[3.4-Dimethoxy-phenyl]- $\beta$-oxo-propionsäure

9) Vgl. W. Wiegrebe, Arch. Pharmaz. 300, 338 (1967).

10) H. Mühlemann und A. Bürgin, Qualitative Arzneimittel-Analyse, 2. Aufl., S. 68, E. Reinhardt-Verlag, München 1956. 
entstanden war; Versuche zu deren Isolierung scheiterten an der geringen Konzentration. Deshalb wurde ein Teil der Säure-Fraktion alkalisiert und erhitzt. Aus diesem Ansatz konnte 9 direkt und als 2.4-Dinitro-phenylhydrazon durch Vergleich mit authentischen Substanzen dünnschichtchromatographisch nachgewiesen werden. Das Massenspektrum bestätigte unseren Befund.

Der Rest der Säure-Fraktion wurde mit Diazomethan verestert. In diesem Ansatz wurde der Methylester von 5 dünnschichtchromatographisch nachgewiesen. Bei weiteren Versuchen konnte $\mathbf{5}$ auch kristallin erhalten werden.

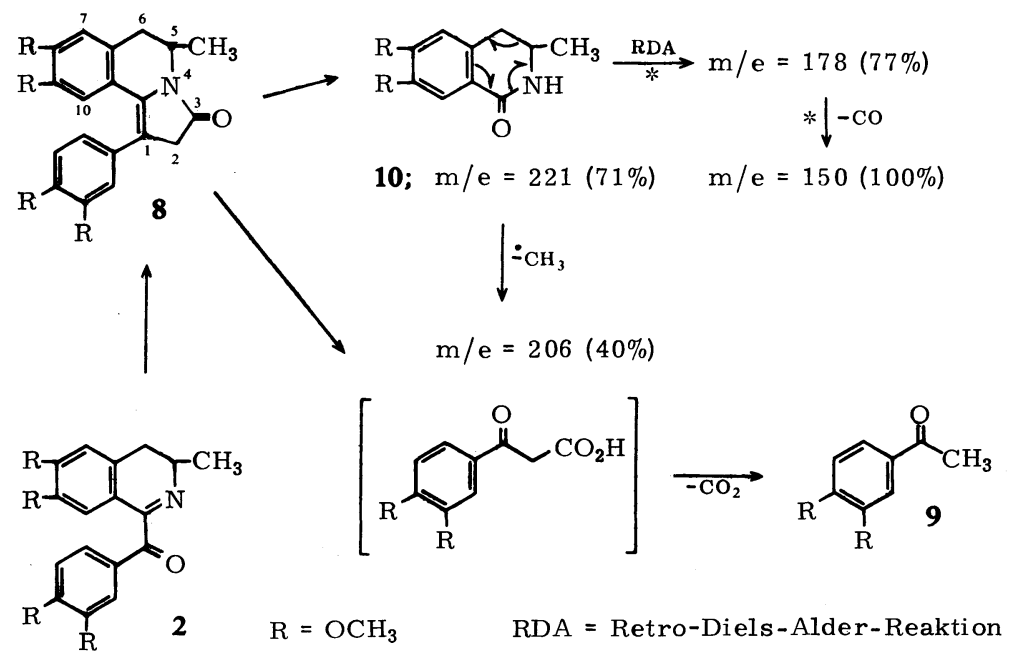

Bei der oxydativen Spaltung der tetrasubstituierten Doppelbindung in 8 sollte neben 9 und 5 auch 3-Methyl-6.7-dimethoxy-1.2.3.4-tetrahydro-isochinolin-1-on (10) entstehen. Wir stellten dieses durch Umsetzung von 1-[3.4-Dimethoxy-phenyl]-2-amino-pro$\operatorname{pan}^{8 \mathrm{~b})}$ mit Chlorameisensäureäthylester und nachfolgenden Ringschluß her. 10 wurde unter den Bedingungen des oxydativen Abbaus nur z. T. zerstört, so daß wir es durch Vergleich mit authentischer Substanz im Dünnschichtchromatogramm der Abbauprodukte von $\mathbf{8}$ lokalisieren, nach Schichtchromatographie kristallin isolieren und durch Vergleich der Massenspektren sowie durch die Mischprobe identifizieren konnten.

Die Absorption des blauvioletten 8 bei $730 \mathrm{~nm}$ kann als Folge eines charge-transferKomplexes aus 2 Molekülen gedeutet werden. Eine Molekulargewichtsbestimmung nach Rast scheiterte, weil 8 sich in der Kampferschmelze unter Rotfärbung zersetzt; die ebullioskopische Bestimmung in Benzol schied wegen der Schwerlöslichkeit von 8 aus. Die osmometrische Bestimmung erbrachte das Molekulargewicht 755. Da sich 
für das Dimere von 8 ein Mol.-Gew. von 790 errechnet, stimmen die Werte hinreichend überein, um das Maximum bei $730 \mathrm{~nm}$ durch die Absorption des angenommenen charge-transfer-Komplexes zu erklären ${ }^{11)}$.

Auch das NMR-Spektrum deutet durch das Auftreten von mehr als 4 Methoxyl-Signalen bzw. mehr als einem $\mathrm{CH}_{3}$-Dublett - in Abhängigkeit voṇ Lösungsmittel und Konzentration - auf einen solchen Komplex hin.

Diese Arbeit wurde durch Forschungsmittel des Landes Niedersachsen gefördert, für die wir auch an dieser Stelle vielmals danken. Herrn Prof. Dr. phil., Dr. med. h. c. H. H. Inhoffen danken wir verbindlich für die Unterstützung dieser Arbeit. Unser Dank gilt auch der Fa. E. Merck, Darmstadt, die diese Arbeit durch .Chemikalienspenden gefördert hat.

\section{Beschreibung der Versuche}

Die Schmelzpunkte wurden im Cu-Block bestimmt und nicht korrigiert. - Präparative Schichtchromatographie (PC): Zur Beschichtung von $20 \times 20 \mathrm{~cm}$-Platten wurden $30 \mathrm{~g}$ Kieselgel $\mathrm{PF}_{254}$ (Merck) mit $75 \mathrm{ccm}$ Wasser angerührt. - Die Spektren wurden mit folgenden Geräten aufgenommen: Leitz-Unicam SP. 200 G (IR), Zeiss-Spektralphotometer PMQ II (UV), Varian HA-100 (NMR; Tetramethylsilan als innerer Standard), MS 9 der Fa. AEI, Manchester (Massenspektren; Ionisierungsenergie $70 \mathrm{eV}$, Quellentemperatur ca. $200^{\circ}$ ).

Umsetzung von 3.4-Dihydro-papaveraldin (2: $\left.R^{1}=H\right)$

$4.0 \mathrm{~g} 2\left(R^{1}=H\right)$ wurden mit $10 \mathrm{ccm}$ Acetanhydrid 10 Min. zu mäßigem Sieden erhitzt. Überschüssiges Acetanhydrid wurde i. Vak. abdestilliert, der Rückstand in möglichst wenig Methanol kalt gelöst und in ca. $100 \mathrm{ccm}$ Wasser gerührt. Die Suspension wurde mit $2 n \mathrm{H}_{2} \mathrm{SO}_{4}$ schwach angesäuert. Es entstand ein flockiger Niederschlag, der abgesaugt, getrocknet, mit Äther gewaschen und aus Methanol umkristallisiert wurde. Ausbeute $400 \mathrm{mg}$ schwachgelbe Nadeln von 3, Schmp. 172 . - UV-Spektrum (Methanol): $\lambda_{\max }(\log \varepsilon)=231$ (4.47), 285 (4.24), $332 \mathrm{~nm}$ (4.25).

$$
\mathrm{C}_{22} \mathrm{H}_{25} \mathrm{NO}_{7} \text { (415.4) Ber. C } 63.60 \mathrm{H} 6.07 \text { N } 3.37 \text { Gef. C } 63.79 \text { H } 5.99 \text { N } 3.23
$$

$30 \mathrm{mg} 3$ wurden mit $3 \mathrm{ccm} 12 \mathrm{n} \mathrm{HCl}$ auf dem siedenden Wasserbad erhitzt. Nach $10 \mathrm{Min}$. war 3 dünnschichtchromatographisch nicht mehr nachweisbar. Nach dem Erkalten wurde mit Wasser verdünnt, mit $\mathrm{NH}_{3}$ alkalisiert und die Base mit Chloroform ausgeschüttelt. Durch Reinigung an $\mathrm{Al}_{2} \mathrm{O}_{3}$ (nach Brockmann) wurde $2\left(\mathrm{R}^{1}=\mathrm{H}\right)$ kristallin erhalten; Schmp. $191^{\circ}$ (Mischprobe). Die IR-Spektren (KBr) waren identisch.

Das Filtrat von 3 wurde mit Äther ausgeschüttelt und diese Ätherphase mit der Ätherlösung vereinigt, die beim Waschen des rohen 3 erhalten worden war (wäßr. Schicht = Basen-Phase). Nach Extrahieren dieser Ätherauszüge mit 1 proz. Natronlauge wurde die wäßr. Phase mit $2 n \mathrm{H}_{2} \mathrm{SO}_{4}$ angesäuert und mit Äther ausgeschüttelt. Im DC mit Benzol/Aceton/Methanol $(6: 2: 2)$ wurde eine Hauptsubstanz neben vielen Verunreinigungen nachgewiesen, die durch PC im gleichen Fließmittel (Laufstrecke $3 \times 20 \mathrm{~cm}$ ) abgetrennt wurde. Die Hauptzone wurde mit Methanol eluiert: Farblose Kristalle vom Schmp. $240^{\circ}$ (Zers.), die in wenig Wasser gelöst und mit $12 n \mathrm{HCl}$ so lange versetzt wurden, bis ein flockiger Niederschlag entstand, der beim

11) Wir danken Herrn Prof. Dr. H. H. Perkampus für die Diskussion dieses Ergebnisses. 
Erwärmen wieder in Lösung ging. Beim Erkalten kristallisierte 5 aus. Ausbeute $7 \mathrm{mg}$, Schmp. $182^{\circ}$ (Lit. ${ }^{12)} 181^{\circ}$ ). -5 wurde in methanol. Lösung mit Diazomethan verestert. Das Produkt war im DC mit Benzol/Methanol $(8: 2)$ identisch mit authent. Ester $\left.{ }^{13}\right)$.

Aus der nach dem Ausschütteln mit Natronlauge verbliebenen Ätherlösung (Neutralstoffe) wurden durch PC mit Benzol/Aceton/Methanol $(6: 1: 3$; Laufstrecke $3 \times 20 \mathrm{~cm})$ die roten Verbindungen $A$ und $B$ sowie 6 vom Schmp. $202^{\circ}$, Ausbeute $11 \mathrm{mg}$, isoliert.

$$
\mathrm{C}_{22} \mathrm{H}_{25} \mathrm{NO}_{5} \text { (383.4) Ber. C } 68.90 \mathrm{H} 6.57 \text { N } 3.65 \text { Gef. C } 68.67 \text { H } 6.72 \text { N } 3.44
$$

Die Basen-Phase (s. S. 125) wurde mit $2 n \mathrm{NH}_{3}$ versetzt und mit $\ddot{A}$ ther extrahiert. Die durch das DC mit Benzol/Aceton/Methanol $(5: 3: 2)$ ausgewiesenen Hauptsubstanzen wurden durch PC mit Benzol/Methanol ( $4: 1$; Laufstrecke $2 \times 20 \mathrm{~cm})$ getrennt. Beide Substanzen kristallisieren aus $\ddot{A}$ thanol. Es handelt sich um $2\left(R^{1}=H\right)$ und 4 , das durch Schmp. $\left(212^{\circ}\right)$, UV- und IR-Spektrum mit authentischer Substanz identifiziert wurde; Ausbeute $14 \mathrm{mg}$.

Umsetzung von 3-Methyl-3.4-dihydro-papaveraldin (2: $\left.\mathrm{R}^{1}=\mathrm{CH}_{3}\right)$

$1.0 \mathrm{~g} 2\left(\mathrm{R}^{1}=\mathrm{CH}_{3}\right)$ wurde, wie $\mathrm{S} .125$ beschrieben, mit $2.5 \mathrm{ccm}$ Acetanhydrid umgesetzt, das Rohprodukt in Chloroform aufgenommen und dünnschichtchromatographisch mit Benzol/Aceton $(7: 3)$ untersucht. Von den vielen Nebenprodukten wurde die grüne Verbindung durch PC mit Benzol/Aceton $(7: 3$; Laufstrecke $3 \times 20 \mathrm{~cm})$ getrennt. Man eluiert die entsprechende Zone mit Chloroform/Methanol (1:1), zieht die Lösungsmittel ab, löst den Rückstand in möglichst wenig Chloroform und filtriert in erwärmtes Cyclohexan. In der Kälte kristallisieren kleine, violett schimmernde Plättchen vom Schmp. 226-228 ${ }^{\circ}$ (Zers.); Ausbeute $100 \mathrm{mg}$ 1-[3.4-Dimethoxy-phenyl]-8.9-dimethoxy-5-methyl-2.3.5.6-tetrahydro-pyrrolo[2.1-a]isochinolin-3-on (8).

Oxydativer Abbau von 8: $100 \mathrm{mg} 8$ wurden in $10 \mathrm{ccm}$ wäßr. Pyridin (50proz.) gelöst, mit $60 \mathrm{mg} \mathrm{KMnO}_{4}$ in $10 \mathrm{ccm}$ des gleichen Lösungsmittels versetzt und $2 \mathrm{Stdn}$. bei Raumtemperatur geschüttelt. Durch Einleiten von $\mathrm{SO}_{2}$ wurde überschüssiges Permanganat reduziert, die nach Filtration klare gelbe Lösung mit $2 n \mathrm{HCl}$ angesäuert und ausgeäthert. In der Ätherphase waren dünnschichtchromatographisch mit Benzol/Aceton/Methanol $(6: 3: 1)$ etwa 10 Substanzen nachweisbar; es wurde mit 5 proz. $\mathrm{NaHCO}_{3}-\mathrm{Lösung}$ ausgeschüttelt, die alkalisch-wäßrige Phase erneut angèsäuert und mit Methylenchlorid extrahiert. In dieser Säuren-Fraktion lag u. a. eine gegenüber 2.4-Dinitro-phenylhydrazin positive Substanz vor, vermutlich $\beta$-[3.4-Dimethoxy-phenyl]- $\beta$-oxo-propionsäure. - Ein Teil der MethylenchloridPhase wurde eingedampft und der Rückstand mit $2 \mathrm{ccm} 5$ proz. Natronlauge $15 \mathrm{Min}$. auf dem siedenden Wasserbad erhitzt. Nach dem Abkühlen wurde mit Äther ausgeschüttelt. Die Ätherlösung enthielt 9, das chromatographisch mit Benzol/Aceton $(9: 1)$ von Begleitstoffen getrennt wurde. In der wäßrigen Phase war keine Säure mehr enthalten, die mit Dinitrophenylhydrazin-Lösung reagierte. - Ein anderer Teil der Methylenchlorid-Phase wurde nach dem Verdampfen des Lösungsmittels mit äther. Diazomethan-Lösung versetzt. Im DC mit Benzol/Methanol $(8: 2)$ fanden wir den Methylester von 5. Bei einem anderen Ansatz wurde die Methylenchloridlösung auf 5 hin aufgearbeitet. Nach
PC mit Benzol/Methanol $(5: 5)$ wurde krist. 5 erhalten.

12) R. L. Shriner, R. C. Fuson und D. Y. Curtin, Systematic Identification of Organic Compounds, S. 279, J. Wiley \& Sons, Inc., New York 1956.

13) K. U. Matsmoto, Ber. dtsch. chem. Ges. 11, 127 (1878). 
Aus dem nach der Reduktion überschüssigen Permanganats erhaltenen Filtrat eines weiteren Reaktionsansatzes wurde 10 durch PC mit Benzol/Aceton $(3: 2)$ abgetrennt und aus Methanol kristallin erhalten; Schmp. $190^{\circ}$, Ausbeute $6 \mathrm{mg}$.

3-Methyl-6.7-dimethoxy-1.2.3.4-tetrahydro-isochinolin-1-on (10). - $5.0 \mathrm{~g}$ Q-Amino-1-[3.4-dimethoxy-phenyl]-propan ${ }^{8 \mathrm{~b})}$ wurden in $50 \mathrm{ccm}$ Chloroform gelöst und mit $50 \mathrm{ccm} 15$ proz. Natronlauge und $5 \mathrm{ccm}$ Chlorameisensäure-äthylester $2 \mathrm{Stdn}$. geschüttelt. Man extrahierte die wäßrige Phase 2 mal mit Äther und die vereinigten organischen Phasen mit $2 n \mathbf{H C l}$. Wir erhielten $6.0 \mathrm{~g} \mathrm{~N}$ - ̈̈thoxycarbonyl-2-amino-1-[3'.4'-dimethoxy-phenyl]-propan als helles Öl, das in $80 \mathrm{ccm}$ Xylol gelöst, mit $2 \mathrm{ccm} \mathrm{POCl}_{3}$ versetzt und $90 \mathrm{Min}$. auf $140^{\circ}$ erhitzt wurde. Die flüchtigen Bestandteile wurden i. Vak. abdestilliert und der Rückstand wurde zunächst mit Wasser, dann mit heißer $2 n \mathrm{HCl}$ ausgezogen. Nach dem Alkalisieren mit $\mathrm{NH}_{3}$ wurde die Lösung mit Chloroform ausgeschüttelt und die Chloroformphase an $\mathrm{Al}_{2} \mathrm{O}_{3}$ (Woelm, neutral, Aktiv.-Stufe 1) chromatographiert. Nach Abdestillieren des Lösungsmittels blieb ein gelbliches Öl zurück, das aus Methanol kristallisierte. Schmp. 190, Ausbeute $600 \mathrm{mg}$.

$$
\mathrm{C}_{12} \mathrm{H}_{15} \mathrm{NO}_{3} \text { (221.3) Ber. C } 65.14 \mathrm{H} 6.83 \text { Gef. C } 65.32 \mathrm{H} 6.81
$$

$N$-Acetyl-tetrahydro-papaverin (7). $-2.8 \mathrm{~g}$ 1.2.3.4-Tetrahydro-papaverin 14 ) wurden mit $30 \mathrm{ccm}$ Acetanhydrid 2 Stdn. unter Rückfluß erhitzt. Das Reaktionsgemisch wurde in $50 \mathrm{ccm}$ Wasser gegossen, die wäßrige Lösung mit $10 \mathrm{ccm} 10$ proz. Salzsäure angesäuert und mit Äther ausgeschüttelt. Das DC mit Benzol/Aceton ( $7: 3)$ zeigte, daß das Tetrahydropapaverin vollständig umgesetzt worden war. 7 kristallisiert aus Äther; Schmp. $115^{\circ}$, Ausbeute $2.5 \mathrm{~g}$. IR (KBr): Breite Bande bei $3415 \mathrm{~cm}^{-1}$. - Massenspektrum ${ }^{15)}$ : Das Molekül-Ion $\mathrm{m} / \mathrm{e}=385$ tritt nur sehr schwach auf; der Peak stärkster Intensität rührt vom $N$-Acetyl-3.4-dihydro6.7-dimethoxy-isochinolinium-Ion $(\mathrm{m} / \mathrm{e}=234)$ her, das Keten abspaltet 4$)(\mathrm{m} / \mathrm{e}=192 ; 57 \%)$. Das Dimethoxybenzyl-Kation $(\mathrm{m} / \mathrm{e}=151)$ tritt mit $5 \%$ Intensität auf.

$$
\mathrm{C}_{22} \mathrm{H}_{27} \mathrm{NO}_{5} \cdot 0.5 \mathrm{H}_{2} \mathrm{O} \text { (394.4) Ber. C } 66.98 \text { H 7.16 Gef. C } 66.63 \mathrm{H} 7.47
$$

14) K. Kindler und W. Peschke, Arch. Pharmaz. 272, 240 (1934).

15) M. Ohashi, J. M. Wilson, H. Budzikiewicz, M. Shamma, W. A. Slusarchyk und C. Djerassi, J. Amer. chem. Soc. 85, 2807 (1963). 